\title{
Start Procedure Action Item
}

National Cancer Institute

\section{Source}

National Cancer Institute. Start Procedure Action Item. NCI Thesaurus. Code C69263.

Indicates the beginning action of a procedure. 